Case Report

\title{
Two Patients with Fulminant Clostridium difficile Enteritis Who Had Not Undergone Total Colectomy: A Case Series and Review of the Literature
}

\author{
Eliza W. Beal, ${ }^{1}$ Rosara Bass, ${ }^{2}$ and Alan E. Harzman ${ }^{1,3}$ \\ ${ }^{1}$ Department of Surgery, The Ohio State University Wexner Medical Center, Columbus, OH 43210, USA \\ ${ }^{2}$ The Ohio State University College of Medicine, Columbus, OH 43210, USA \\ ${ }^{3}$ Division of Colorectal Surgery, Department of Surgery, The Ohio State University Wexner Medical Center, Columbus, OH 43210, USA \\ Correspondence should be addressed to Eliza W. Beal; eliza.beal@osumc.edu
}

Received 9 July 2015; Revised 1 November 2015; Accepted 2 November 2015

Academic Editor: Boris Kirshtein

Copyright (C) 2015 Eliza W. Beal et al. This is an open access article distributed under the Creative Commons Attribution License, which permits unrestricted use, distribution, and reproduction in any medium, provided the original work is properly cited.

\begin{abstract}
Introduction. Clostridium difficile is the most common cause of healthcare associated infectious diarrhea, and its most common clinical manifestation is pseudomembranous colitis. Small bowel enteritis is reported infrequently in the literature and typically occurs only in patients who have undergone ileal pouch anastomosis due to inflammatory bowel disease or total abdominal colectomy for other reasons. Presentation of Cases. We report here two cases in which patients developed small bowel C. difficile enteritis in the absence of these underlying conditions. Discussion. Neither patient had underlying inflammatory bowel disease and both had a significant amount of colon remaining. Conclusion. These two cases demonstrate that small bowel C. difficile enteritis should be included in the differential diagnosis of patients on antibiotic therapy who demonstrate signs and symptoms of worsening abdominal disease during their postoperative course, even if they lack the major predisposing factors of inflammatory bowel disease or history of total colectomy.
\end{abstract}

\section{Introduction}

Clostridium difficile is the most common cause of healthcare associated infectious diarrhea [1]. Symptoms and signs of severe C. difficile infection include watery, foul-smelling diarrhea, pseudomembranous colitis, marked peripheral leukocytosis, acute renal failure, and hypotension [2]. Exposure to antibiotics is the major risk factor for $C$. difficile. The incidence of $C$. difficile has increased significantly over the past 25 years with a concomitant increase in severity and mortality [2]. Intravenous metronidazole and oral vancomycin remain the treatments of choice for $C$. difficile colitis [2] and were long considered equivalent [3]. In a prospective, randomized, placebo-controlled trial it was demonstrated that the two agents had similar efficacy in mild infection, but that vancomycin had a significantly higher response rate in patients with severe infection $(97 \%$ versus $76 \%, P=$ 0.02) [3]. Other studies have demonstrated similar results [4]. Other agents currently being evaluated include difimicin and tolevamer [2]. Small bowel enteritis is reported infrequently in the literature and is typically reported only in patients with inflammatory bowel disease (IBD) who have undergone ileal pouch anal anastomosis [5] or who have undergone total abdominal colectomy for other reasons [6]. The authors who have written on the topic seem to agree that early diagnosis is important in obtaining improved outcomes.

\section{Presentation of Cases}

2.1. Case 1. A 63-year-old female presented for a right hemicolectomy and umbilical hernia repair for a large transverse colon polyp found on screening colonoscopy. She received 1.0 gram of ertapenem preoperatively. On postoperative day four she developed abdominal pain and distention, nausea, vomiting, and diarrhea. A nasogastric tube was placed which drained two liters of fluid. Computed tomography (Figure 1) demonstrated dilated small bowel loops with a small amount of gas in the colon consistent with an ileus. She developed hypotension, tachycardia, and reduced oxygen saturation and was transferred to the surgical intensive care unit 

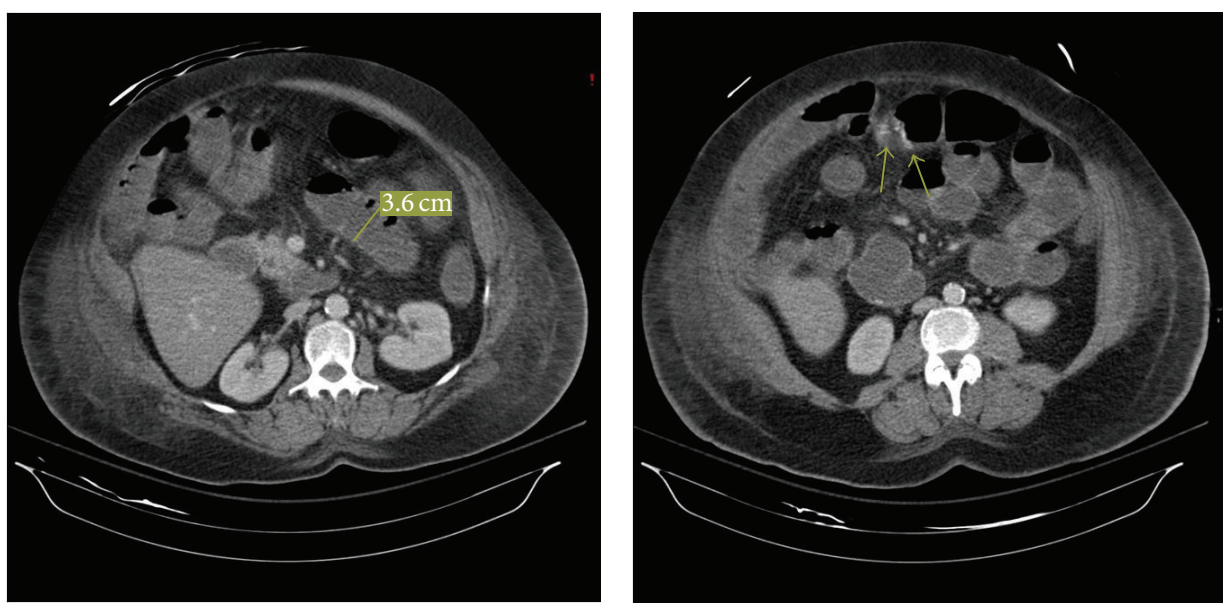

FIGURE 1: Computed tomography of the abdomen and pelvis with IV and PO contrast demonstrated diffuse dilation and fluid filling in the small bowel to the level of the ileocolic anastomosis out of proportion to the large bowel gas concerning for low-grade small bowel obstruction versus postoperative ileus.

(SICU) on mechanical ventilation and vasopressor support. She developed acute kidney failure. She was started on continuous venovenous hemodialysis to assist in metabolite and volume control and empiric broad spectrum antibiotics. On postoperative day five she was taken to the operating room for exploratory laparotomy. At the time of reexploration there was no acute intra-abdominal process noted, and her anastomosis was intact. She was transferred back to the SICU where she required increasing vasopressor support and continued mechanical ventilation. Flexible sigmoidoscopy was performed to the descending colon, showing no pseudomembrane formation nor other abnormalities. On postoperative day seven she had a cardiac arrest and expired despite attempted resuscitation. An autopsy was performed which demonstrated pseudomembranous enteritis throughout her small bowel with patchy transmural inflammation and hemorrhage and focal areas of necrosis. Opening of the small bowel demonstrated pseudomembranous exudates, which filled the lumen. The colon did not show pseudomembranes. The pathology of the resected mass demonstrated welldifferentiated adenocarcinoma of the colon.

2.2. Case 2. A 63-year-old female presented with pelvic abscess and rectovaginal fistula several months after undergoing a low anterior resection at another institution complicated by intra-abdominal sepsis from dehiscence of her anastomosis. This had been treated with diverting loop ileostomy and closure of her abdominal fascia with biologic mesh. Since that time she had developed purulent drainage from her vagina and rectum. She had been started on per oral amoxicillin-clavulanate. She was taken to the operating room for low anterior resection with coloanal anastomosis, small bowel resection, primary repair of parastomal hernia with mesh, and drain placement. The patient was on broad spectrum antibiotics at the time of surgery. Specific preoperative antibiotics were not given. On postoperative day three she developed nausea and vomiting. On postoperative day four she developed hypotension. A nasogastric tube was placed with return of bilious output. She became hypotensive and was transferred to the surgical intensive care unit (SICU) for fluid resuscitation. She was afebrile, with moderate abdominal tenderness, reduced urine output, and elevated creatinine. The decision was made to proceed to the operating room. At the time of reoperation her anastomoses were intact, but the abdomen could not be closed. An ABThera Open Abdomen Negative Pressure Wound Therapy System was placed and she was returned to the SICU where she was started on intravenous metronidazole and oral vancomycin due to concern for small bowel $C$. difficile enteritis after pseudomembranes were noted on the ileostomy. A C. difficile toxin assay was positive at that time. Red rubber catheters were placed in both sides of her ileostomy and her rectum taking care not to disrupt her coloanal anastomosis. $500 \mathrm{mg}$ of vancomycin was placed in each of these tubes and down her nasogastric tube every 6 hours.

Her hospital course was further complicated by ventricular tachycardia, heart failure intolerance of enteral feeds, cholestatic jaundice, need for reoperation for placement of absorbable mesh for closure of the abdominal cavity, leukocytosis, need for total parenteral nutrition (TPN), tracheostomy, development of enterocutaneous fistula, multiple intraabdominal abscesses, a repeat episode of $C$. difficile enteritis, atrial fibrillation with rapid ventricular rate, and a urinary tract infection. She remained in the hospital for two months.

At the time of discharge, her tracheostomy had been removed; she was tolerating snacks of thickened liquids, had a nasoenteric feeding tube in place, and was receiving TPN and intravenous metronidazole. A wound manager was being used to control her fistula output. The fistula subsequently closed, and she remains healthy but with a large ventral hernia.

\section{Discussion and Review of the Literature}

Clinical manifestations of severe $C$. difficile infection include watery, foul-smelling diarrhea, pseudomembranous colitis, 
TABle 1: Demographic features, risk factors, and outcomes of reviewed case reports. ${ }^{*} \mathrm{IBD}=$ inflammatory bowel disease.

\begin{tabular}{|c|c|c|}
\hline$N=63$ cases & Number & $\begin{array}{c}\text { Percent/standard } \\
\text { deviation }\end{array}$ \\
\hline Crohn's disease & 5 & $7.9 \%$ \\
\hline Ulcerative colitis & 27 & $42.9 \%$ \\
\hline No IBD* & 31 & $49.2 \%$ \\
\hline Median age & 53.1 & 20.8 \\
\hline \multicolumn{3}{|l|}{ Received antibiotics } \\
\hline Yes & 55 & $87.3 \%$ \\
\hline No & 7 & $11.1 \%$ \\
\hline Not reported & 1 & $1.6 \%$ \\
\hline \multicolumn{3}{|l|}{ Antibiotic type } \\
\hline Cephalosporin & 19 & $30.2 \%$ \\
\hline $\begin{array}{l}\text { Penicillin/penicillin } \\
\text { derivatives }\end{array}$ & 12 & $19.1 \%$ \\
\hline Fluoroquinolone & 13 & $20.6 \%$ \\
\hline Aminoglycoside & 5 & $7.9 \%$ \\
\hline Metronidazole & 14 & $22.2 \%$ \\
\hline Carbapenem & 2 & $3.2 \%$ \\
\hline Vancomycin & 4 & $6.4 \%$ \\
\hline Rifampin & 1 & $1.6 \%$ \\
\hline $\begin{array}{l}\text { Patients with total } \\
\text { proctocolectomy with ileal pouch } \\
\text { anal anastomosis }\end{array}$ & 26 & $41.3 \%$ \\
\hline \multicolumn{3}{|l|}{ Outcome } \\
\hline Resolution & 44 & $69.8 \%$ \\
\hline Death & 19 & $30.1 \%$ \\
\hline
\end{tabular}

marked peripheral leukocytosis, acute renal failure, and hypotension [2] with exposure to antibiotics as the major risk factor. Treatment for $C$. difficile infection includes intravenous metronidazole and oral vancomycin [2], which were long considered equivalent [3]. More recent studies have refuted this, suggesting that patients with severe $C$. difficile infection respond better to oral vancomycin $[3,4]$. Other agents currently being investigated include difimicin and tolevamer [2]. Small bowel enteritis is reported infrequently in the literature and is typically reported only in patients with inflammatory bowel disease (IBD) who have undergone ileal pouch anal anastomosis [5] or who have undergone total abdominal colectomy for other reasons [6].

A literature review was performed. PubMed was queried using the terminology "Clostridium difficile enteritis." There were 215 entries returned. These were examined for articles reporting cases of $C$. difficile enteritis. The references of these articles were also examined for additional cases. There were sixty-six cases reported in the literature between 1980 and 2013 (Table 1). Two cases were excluded due to the full-text article being in Dutch [7] and Spanish [8]. One case was excluded because full text of the article could not be located [9]. In total sixty-three cases were included in our analysis.

Of the 63 cases identified in the literature, thirty-one (49.2\%) patients did not have a diagnosis of inflammatory bowel disease (IBD). Twenty-seven (42.8\%) patients had ulcerative colitis and five (7.9\%) patients had Crohn's disease. Fifty-five of the $63(87.3 \%)$ patients received antibiotics in the time period preceding their development of $C$. difficile enteritis. Many of the patients received multiple antibiotics with 19 (30.2\%) receiving cephalosporins, 12 (19.1\%) receiving penicillin or penicillin derivatives, $13(20.6 \%)$ receiving fluoroquinolone, 5 (7.9\%) receiving aminoglycoside, 14 (22.2\%) receiving metronidazole, 2 (3.2\%) receiving carbapenem antibiotic, 4 (6.4\%) receiving cotrimoxazole, 4 (6.4\%) receiving vancomycin, and $1(1.6 \%)$ receiving rifampin. Twenty-six (41\%) of the patients had previously undergone a total proctocolectomy with ileal pouch anal anastomosis or total colectomy with end ileostomy for medically refractory ulcerative colitis. Thirty-two (50.8\%) of the patients in this study had no remaining colon at the time they were diagnosed with C. difficile enteritis. Forty-four (69.8\%) of the patients experienced resolution of their symptoms and $19(30.2 \%)$ died [1, 10-47].

In keeping with our analysis, in a recent systematic review of the literature, Killeen et al. noted that the majority of reported cases occurred after colonic resection and that IBD in and of itself may predispose patients to $C$. difficile enteritis [48].

\section{Conclusion}

The patients presented here had several features in common. Both underwent surgery that altered their intestinal anatomy. In the first case this was a right hemicolectomy for colon cancer and in the second it was a redo low anterior resection. What is particularly interesting is that neither patient had underlying inflammatory bowel disease and both had a significant amount of colon remaining. While this is true of over half of the patients in the literature, the presence of pseudomembranes in the colon meant that, for one patient, this diagnosis was only accomplished during the autopsy. For the other a $C$. difficile toxin was isolated from the ileostomy output and pseudomembranes were visible on the stoma.

Many authors seem to agree that early diagnosis and treatment are keys to surviving $C$. difficile enteritis. Because this is a rare condition, especially in patients without underlying inflammatory bowel disease or history of total colectomy, the first patient was not diagnosed with $C$. difficile enteritis until autopsy. Our experience with this patient led to a higher index of suspicion in the second case, which allowed early identification of $C$. difficile. The timely treatment allowed the survival of this patient. These two cases demonstrate that small bowel $C$. difficile enteritis should be included in the differential diagnosis of patients on antibiotic therapy who demonstrate signs and symptoms of worsening abdominal disease during their postoperative course, even if they lack the major predisposing factors of inflammatory bowel disease or history of total colectomy.

\section{Consent}

A written informed consent was obtained from the patients or next of kin for publication of this case report and accompanying images. 


\section{Conflict of Interests}

The authors declare that there is no conflict of interests.

\section{Authors' Contribution}

Eliza W. Beal, M.D., Rosara Bass, M.D., and Dr. Alan E. Harzman were all involved in the conception, design, literature review, analysis of data, paper preparation, revision, and approval of the final paper.

\section{References}

[1] S. P. Dineen, S. H. Bailey, T. H. Pham, and S. Huerta, "Clostridium difficile enteritis: a report of two cases and systematic literature review," World Journal of Gastrointestinal Surgery, vol. 5, no. 3, pp. 37-42, 2013.

[2] C. P. Kelly and J. T. LaMont, "Clostridium difficile-more difficult than ever," The New England Journal of Medicine, vol. 359, no. 18, pp. 1932-1940, 2008.

[3] F. A. Zar, S. R. Bakkanagari, K. M. L. S. T. Moorthi, and M. B. Davis, "A comparison of vancomycin and metronidazole for the treatment of Clostridium difficile-associated diarrhea, stratified by disease severity," Clinical Infectious Diseases, vol. 45, no. 3, pp. 302-307, 2007.

[4] T. Louie, M. Gerson, D. Grimard, S. Johnson, A. Poirier, and J. Peppe, "Results of a phase III trial comparing tolevamer, vancomycin and metronidazole in patients with Clostridium difficile-associated diarrhea (CDI)," in Proceedings of the 47th Annual Interscience Conference on Antimicrobial Agents and Chemotherapy, Chicago, Ill, USA, September 2007.

[5] Y. Li, J. Qian, E. Queener, and B. Shen, "Risk factors and outcome of PCR-detected Clostridium difficile infection in ileal pouch patients," Inflammatory Bowel Diseases, vol. 19, no. 2, pp. 397-403, 2013.

[6] A. Tsiouris, J. A. Neale, C. A. Reickert, and M. Times, “Clostridium difficile of the ileum following total abdominal colectomy, with or without proctectomy: who is at risk?" Diseases of the Colon and Rectum, vol. 55, no. 4, pp. 424-428, 2012.

[7] M. Wiggelinkhuizen and M. A. N. Gerrits, "Clostridium difficile-induced necrotizing enteritis," Nederlands Tijdschrift voor Geneeskunde, vol. 155, no. 49, Article ID A2414, 2011.

[8] A. R. Martínez, Y. R. Pizarro, F. M. Arrieta et al., "Clostridium difficile enteritis," Gastroenterologia y Hepatologia, vol. 34, no. 8, pp. 539-545, 2011 (Spanish).

[9] V. E. Pricolo, S. Chao, and A. Gregorian, "Clostridium difficile pouchitis after proctocolectomy with ileal pouch-anal anastomosis," American Surgeon, vol. 77, no. 11, pp. E214-E215, 2011.

[10] S. Hariri, P. Gouin, J.-J. Tuech, B. Veber, and B. Dureuil, "Clostridium difficile infection causing multiple organ failure and small-bowel enteritis," Clinics and Research in Hepatology and Gastroenterology, vol. 35, no. 2, pp. 142-144, 2011.

[11] C. Holmer, U. Zurbuchen, B. Siegmund, U. Reichelt, H. J. Buhr, and J.-P. Ritz, "Clostridium difficile infection of the small bowel-two case reports with a literature survey," International Journal of Colorectal Disease, vol. 26, no. 2, pp. 245-251, 2011.

[12] L. E. Kurtz, S. S. Yang, and S. Bank, "Clostridium difficileassociated small bowel enteritis after total proctocolectomy in a Crohn's disease patient," Journal of Clinical Gastroenterology, vol. 44, no. 1, pp. 76-77, 2010.
[13] K. Thomas, J. Taylor, L. Everitt, and R. Nelson, "Clostridium difficile does not only affect the colon: a case series," Colorectal Disease, vol. 13, no. 6, pp. e156-e157, 2011.

[14] D. Gagandeep and S. Ira, "Clostridium difficile enteritis 9 years after total proctocolectomy: a rare case report," The American Journal of Gastroenterology, vol. 105, no. 4, pp. 962-963, 2010.

[15] M. S. Khan, D. Levy, and S. Mann, "Clostridium difficile infection in the absence of a colon," BMJ Case Reports, 2010.

[16] M. W. Causey, M. P. Spencer, and S. R. Steele, "Clostridium difficile enteritis after colectomy," American Surgeon, vol. 75, no. 12, pp. 1203-1206, 2009.

[17] B. Shen, F. H. Remzi, and V. W. Fazio, "Fulminant Clostridium difficile-associated pouchitis with a fatal outcome," Nature Reviews Gastroenterology and Hepatology, vol. 6, no. 8, pp. 492495, 2009.

[18] C. Lavallée, B. Laufer, J. Pépin, A. Mitchell, S. Dubé, and A.C. Labbé, "Fatal Clostridium difficile enteritis caused by the BI/NAP1/027 strain: a case series of ileal C. difficile infections," Clinical Microbiology and Infection, vol. 15, no. 12, pp. 1093-1099, 2009.

[19] B. Wee, J. A. D. Poels, I. J. Mccafferty, P. Taniere, and J. Olliff, "A description of CT features of Clostridium difficile infection of the small bowel in four patients and a review of literature," British Journal of Radiology, vol. 82, no. 983, pp. 890-895, 2009.

[20] R. N. Williams, D. Hemingway, and A. S. Miller, "Enteral Clostridium difficile, an emerging cause for high-output ileostomy," Journal of Clinical Pathology, vol. 62, no. 10, pp. 951-953, 2009.

[21] E. Boland and J. S. Thompson, "Fulminant Clostridium difficile enteritis after proctocolectomy and ileal pouch-anal anastamosis," Gastroenterology Research and Practice, vol. 2008, Article ID 985658, 5 pages, 2008.

[22] O. Peacock, W. Speake, A. Shaw, and A. Goddard, "Clostridium difficile enteritis in a patient after total proctocolectomy," BMJ Case Reports, vol. 2009, Article ID 1165, 2009.

[23] F. Fleming, N. Khursigara, N. O’Connell, S. Darby, and D. Waldron, "Fulminant small bowel enteritis: a rare complication of Clostridium difficile-associated disease," Inflammatory Bowel Diseases, vol. 15, no. 6, pp. 801-802, 2009.

[24] F. A. Yafi, C. R. Selvasekar, and R. R. Cima, "Clostridium difficile enteritis following total colectomy," Techniques in Coloproctology, vol. 12, no. 1, pp. 73-74, 2008.

[25] K. E. Follmar, S. A. Condron, I. I. Turner, J. D. Nathan, and K. A. Ludwig, "Treatment of metronidazole-refractory Clostridium difficile enteritis with vancomycin," Surgical Infections, vol. 9, no. 2, pp. 195-200, 2008.

[26] M. S. H. El Muhtaseb, J. K. Apollos, and J. S. Dreyer, "Clostridium difficile enteritis: a cause for high ileostomy output," ANZ Journal of Surgery, vol. 78, no. 5, p. 416, 2008.

[27] M. J. Wood, N. Hyman, J. C. Hebert, and H. Blaszyk, "Catastrophic Clostridium difficile enteritis in a pelvic pouch patient: report of a case," Journal of Gastrointestinal Surgery, vol. 12, no. 2, pp. 350-352, 2008.

[28] K. A. Kim, P. Wry, E. Hughes Jr., J. Butcher, and D. Barbot, "Clostridium difficile small-bowel enteritis after total proctocolectomy: a rare but fatal, easily missed diagnosis. Report of a case," Diseases of the Colon and Rectum, vol. 50, no. 6, pp. 920 923, 2007.

[29] S. J. Lundeen, M. F. Otterson, D. G. Binion, E. T. Carman, and W. J. Peppard, "Clostridium difficile enteritis: an early postoperative complication in inflammatory bowel disease patients after 
colectomy," Journal of Gastrointestinal Surgery, vol. 11, no. 2, pp. 138-142, 2007.

[30] B. Shen, J. R. Goldblum, T. L. Hull, F. H. Remzi, A. E. Bennett, and V. W. Fazio, "Clostridium difficile-associated pouchitis," Digestive Diseases and Sciences, vol. 51, no. 12, pp. 2361-2364, 2006.

[31] F. D. Hayetian, T. E. Read, M. Brozovich, R. P. Garvin, and P. F. Caushaj, "Ileal perforation secondary to Clostridium difficile enteritis: report of 2 cases," Archives of Surgery, vol. 141, no. 1, pp. 97-99, 2006.

[32] S. D. Mann, J. Pitt, R. G. Springall, and A. V. Thillainayagam, "Clostridium difficile infection-an unusual cause of refractory pouchitis: report of a case," Diseases of the Colon and Rectum, vol. 46, no. 2, pp. 267-270, 2003.

[33] J. F. Freiler, S. J. Durning, and P. T. Ender, "Clostridium difficile small bowel enteritis occurring after total colectomy," Clinical Infectious Diseases, vol. 33, no. 8, pp. 1429-1432, 2001.

[34] J. J. Tjandra, A. Street, R. J. Thomas, R. Gibson, P. Eng, and J. Cade, "Fatal Clostridium difficile infection of the small bowel after complex colorectal surgery," ANZ Journal of Surgery, vol. 71, no. 8, pp. 500-503, 2001.

[35] A. Jacobs, K. Barnard, R. Fishel, and J. D. Gradon, "Extracolonic manifestations of Clostridium difficile infections. Presentation of 2 cases and review of the literature," Medicine, vol. 80, no. 2, pp. 88-101, 2001.

[36] Z. Vesoulis, G. Williams, and B. Matthews, "Pseudomembranous enteritis after proctocolectomy: report of a case," Diseases of the Colon and Rectum, vol. 43, no. 4, pp. 551-554, 2000.

[37] K. A. Kralovich, J. Sacksner, R. A. Karmy-Jones, and J. C. Eggenberger, "Pseudomembranous colitis with associated fulminant ileitis in the defunctionalized limb of a jejunal-ileal bypass: report of a case," Diseases of the Colon and Rectum, vol. 40, no. 5, pp. 622-624, 1997.

[38] C. C. M. Boey, T. M. Ramanujam, and L. M. Looi, "Clostridium difficile-related necrotizing pseudomembranous enteritis in association with Henoch-Schonlein purpura," Journal of Pediatric Gastroenterology and Nutrition, vol. 24, no. 4, pp. 426429, 1997.

[39] H. F. Yee Jr., R. S. Brown Jr., and J. W. Ostroff, "Fatal Clostridium difficile enteritis after total abdominal colectomy," Journal of Clinical Gastroenterology, vol. 22, no. 1, pp. 45-47, 1996.

[40] B. Tsutaoka, J. Hansen, D. Johnson, and M. Holodniy, "Antibiotic-associated pseudomembranous enteritis due to Clostridium difficile," Clinical Infectious Diseases, vol. 18, no. 6, pp. 982-984, 1994.

[41] D. P. Kuntz, M. J. Shortsleeve, P. A. Kantrowitz, and G. P. Gauvin, "Clostridium difficile enteritis. A cause of intramural gas," Digestive Diseases and Sciences, vol. 38, no. 10, pp. $1942-$ 1944, 1993.

[42] D. L. Miller, J. D. Sedlack, and R. W. Holt, "Perforation complicating rifampin-associated pseudomembranous enteritis," Archives of Surgery, vol. 124, no. 9, p. 1082, 1989.

[43] G. P. Testore, A. Pantosti, and G. Panichi, "Pseudomembranous enteritis associated with Clostridium difficile," Italian Journal of Gastroenterology, vol. 16, no. 3, pp. 229-230, 1984.

[44] J. R. Shortland, R. C. Spencer, and J. L. Williams, "Pseudomembranous colitis associated with changes in an ileal conduit," Journal of Clinical Pathology, vol. 36, no. 10, pp. 1184-1187, 1983.

[45] R. H. Taylor, S. P. Borriello, and A. J. Taylor, "Isolation of Clostridium difficile from the small bowel," British Medical Journal, vol. 283, article 412, 1981.
[46] J. S. Hyams, M. M. Berman, and H. Helgason, "Nonantibioticassociated enterocolitis caused by Clostridium difficile in an infant," The Journal of Pediatrics, vol. 99, no. 5, pp. 750-752, 1981.

[47] J. T. LaMont, Y. M. Trnka, J. T. Lamont, and Y. M. Trnka, "Therapeutic implications of Clostridium difficile toxin during relapse of chronic inflammatory bowel disease," The Lancet, vol. 315, no. 8165, pp. 381-383, 1980.

[48] S. Killeen, S. T. Martin, J. Hyland, P. R. O’ Connell, and D. C. Winter, "Clostridium difficile enteritis: a new role for an old foe," Surgeon, vol. 12, no. 5, pp. 256-262, 2014. 


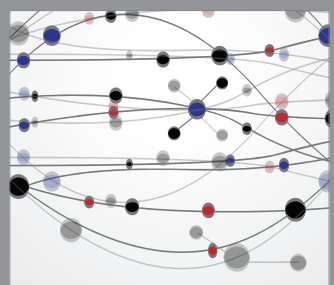

The Scientific World Journal
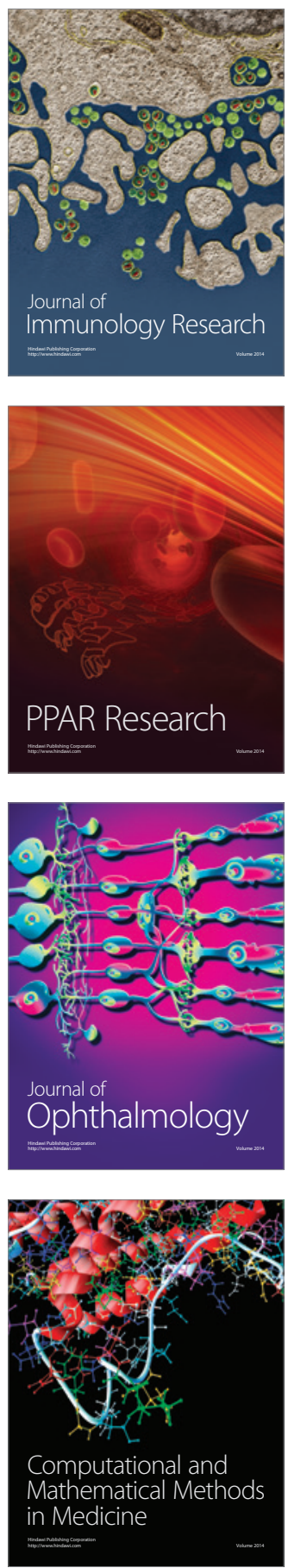

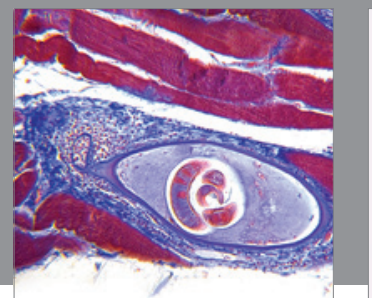

Gastroenterology

Research and Practice
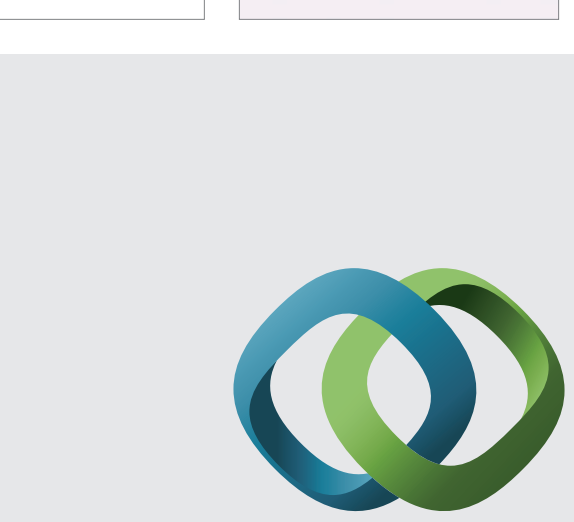

\section{Hindawi}

Submit your manuscripts at

http://www.hindawi.com
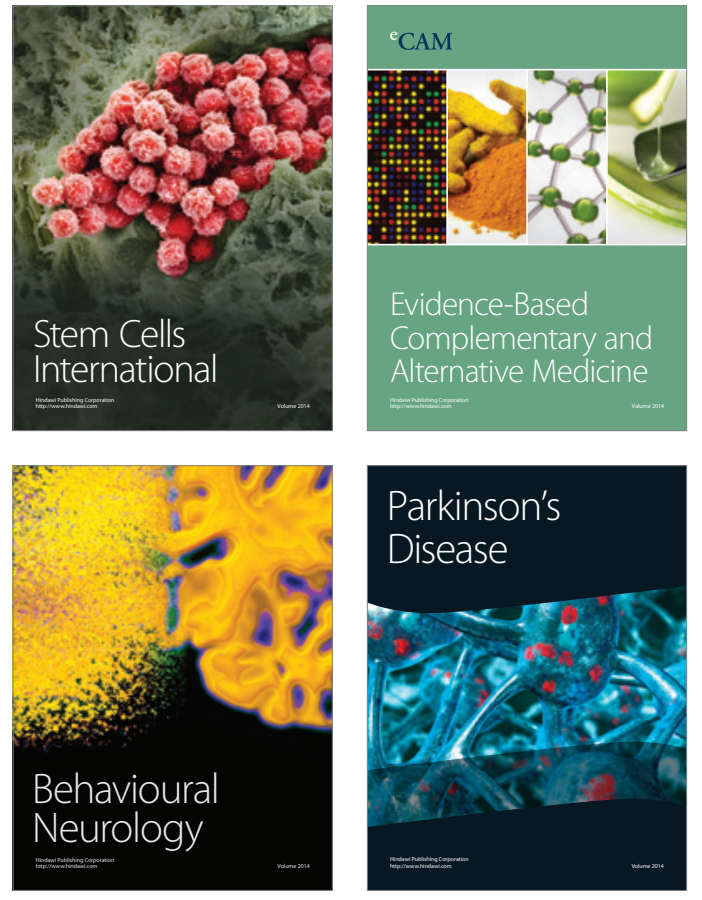
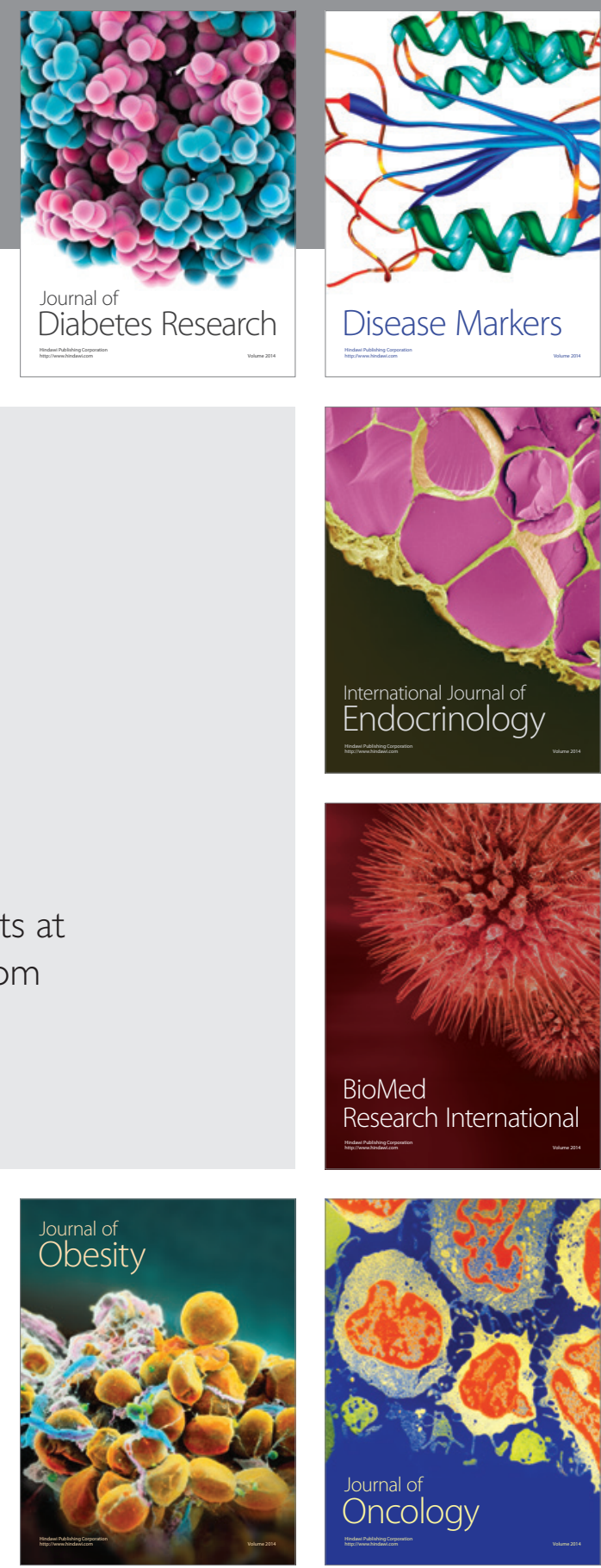

Disease Markers
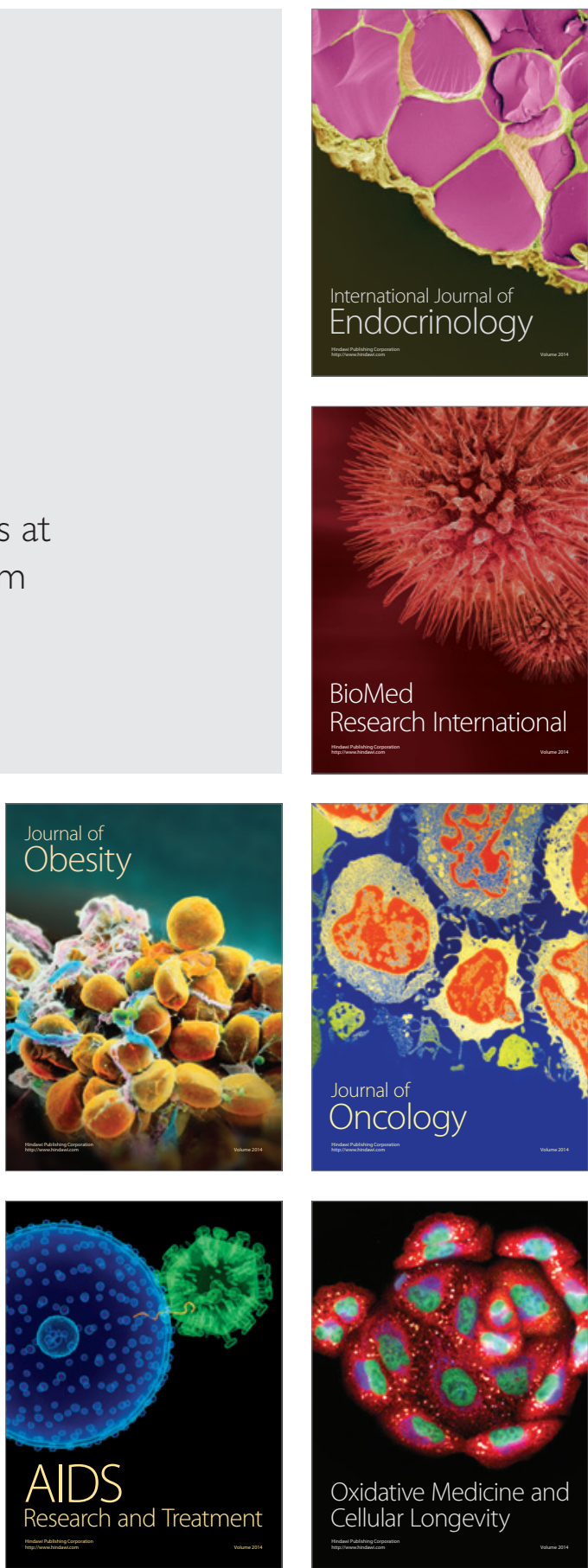